\section{Severe Headache in Emergency Room: Migraine or Digital Eye Strain}

Among primary headaches in children, tension type headache and migraine form the most common causes of headache. With the increasing use of digital devices globally, digital eye strain (DES) or computer vision syndrome (CVS) has been increasing, with the 2016 digital eye strain report documenting a self-reported prevalence of nearly $65 \%$ [1]. Headache has been considered to be one of the five most common symptoms associated with DES according to American Optometric Association [2].

A 14-year-old girl presented to the emergency room with the complaints of severe bitemporal headache with heaviness in eyes, vomiting and undocumented fever for past 15 days. The headache was severe enough to hinder studies, and she had to quit her online examinations due to the headache. Vitals of the patient were within normal ranges and she was afebrile during hospital stay. No signs of meningeal irritation were present. Fundus evaluation was normal. Lumbar puncture and magnetic resonance imaging of brain were done to rule out causes of secondary headache, and were found to be normal. A provisional diagnosis of migraine without aura was made but there was neither previous history of such attacks nor any positive family history. Since the girl had a history of watering of eyes while watching television (TV), an ophthalmic evaluation was performed that revealed dry eyes and a refractory error of $-0.25 \mathrm{D}$ in both eyes. On further detailed history, it was found that the adolescent was having a screen time of 7 hours daily for past 10 months (4 hours of online classes on smartphone due to the pandemic and 3 hours of TV watching). A computer vision syndrome questionnaire (CVSQ) [3] was used to rule out digital eye strain as the cause of headache, and the total score was found to be 18 indicating severe CVS. Initially the patient was given oral analgesics and was advised to have a reduced screen time for next 4 weeks. After one week, the analgesics were stopped. Presently the patient is asymptomatic.

Educational screen use, with appropriate precautions, was advised. The symptomatology of DES or CVS can be related to extraocular, ocular surface or accommodative mechanism leading to severe headache [4]. So objective visual assessment of such patients should not be limited to the assessment of refractory error alone but should also include an orthoptic vision screening for detecting errors of accommodation including unilateral and alternate cover and uncover tests at near vision [5]. Even small aberrations in these tests can lead to symptoms, and may continue progressing uncorrected.

SANDhya Chauhan* AND Himanshu GaRg Department of Pediatrics, Sri Ram Murti Smarak Institute of Medical Sciences, Bareilly, Uttar Pradesh.

\section{REFERENCES}

*drsandhyapedia@gmail.com

1. Sheppard AL, Wolffsohn JS. Digital eye strain: Prevalence, measurement and amelioration. BMJ Open Ophthalmol. 2018; 3:e000146.

2. American Optometric Association. Computer vision syndrome 2017. Available from: https://www.aoa.org/patients-and-public/ caring-for-your-vision/protecting-your-vision/computer-visionsyndrome? sso $=y$

3. Seguí MdM, Cabrero-García J, Crespo A, et al. A reliable and valid questionnaire was developed to measure computer vision syndrome at the workplace. J Clinical Epidemiol. 2015;68:662-73.

4. KY Loh, Redd SC. Understanding and preventing computer vision syndrome. Malays Fam Physician. 2008;3:128-30.

5. Wallace DK, Morse CL, Melia M, et al. Pediatric eye evaluations preferred practice pattern: I. Vision screening in the primary care and community setting; II. Comprehensive ophthalmic examination. Ophthalmology. $2018 ; 125: 184-227$.

\section{Non-availability of Parenteral Preparations of Vitamin A: Is a Silent Surge of Bronchopulmonary Dysplasia Happening in India?}

Bronchopulmonary dysplasia (BPD) continues to be one of the most important challenges in the care of the preterm infants, affecting approximately one-quarter of very low birth weight infants [1]. With better availability and improved quality of care of neonatal intensive care units (NICUs) in India, more and more such babies are surviving. As vitamin A is accumu-lated mainly in the third trimester, preterm infants may have low vitamin A levels at birth, which may contribute to an increased risk of developing BPD. With the large number of preterm babies surviving, there is possibility of an increase in number of BPD cases.

Most of randomized trials to study efficacy of vitamin A supplementation to prevent BPD used parenteral preparations. Globally, trials testing efficacy of oral vitamin A supplemen-tation in preventing BPD has not shown its role [2].

During the last few years there is increasing difficulty in getting intramuscular preparations of vitamin A. Thus currently 